Check for updates

Cite this: RSC Adv., 2020, 10, 17336

Received 31st December 2019

Accepted 19th April 2020

DOI: $10.1039 / c 9 r a 11056 a$

rsc.li/rsc-advances

\title{
A dopamine electrochemical sensor based on a platinum-silver graphene nanocomposite modified electrode $\uparrow$
}

\author{
Nadzirah Sofia Anuar, (iD *a Wan Jeffrey Basirun, ${ }^{* a b}$ Md. Shalauddin ${ }^{a b}$ \\ and Shamima Akhter ${ }^{a}$
}

A platinum-silver graphene ( $\mathrm{Pt}-\mathrm{Ag} / \mathrm{Gr}$ ) nanocomposite modified electrode was fabricated for the electrochemical detection of dopamine (DA). Electrochemical studies of the Pt-Ag/Gr nanocomposite towards DA detection were performed by cyclic voltammetry (CV) and differential pulse voltammetry (DPV). The CV analysis showed that Pt-Ag/Gr/GCE had enhanced electrocatalytic activity towards DA oxidation due to the synergistic effects between the platinum-silver nanoparticles and graphene. The DPV results showed that the modified sensor demonstrated a linear concentration range between 0.1 and $60 \mu \mathrm{M}$ with a limit of detection of $0.012 \mu \mathrm{M}$. The Pt-Ag/Gr/GCE presented satisfactory results for reproducibility, stability and selectivity. The prepared sensor also showed acceptable recoveries for a real sample study.

\section{Introduction}

Dopamine (DA), a neurochemical transmitter in the brain was discovered by Arvid Carlsson in 1957. ${ }^{1}$ Dopamine (DA) affects the cardiovascular, central nervous, endocrine and renal systems. ${ }^{2}$ DA also controls certain physiological conditions such as attention, learning, memory, movement, mood, behaviour and mental cognition. ${ }^{3,4}$ Imbalance in DA levels in the human brain may cause depression, addiction, schizophrenia and neurodegenerative diseases such as Alzheimer's and Parkinson's. ${ }^{3,5}$ Thus, the construction of a facile, selective and sensitive method for DA detection is necessary for monitoring DA levels in the human body. Several methods have been employed for the determination of DA such as chemiluminescence, chromatography, colorimetric, fluorescence and spectrophotometry. ${ }^{6-8}$ However, these conventional methods involve tedious sample pre-treatments, expensive and are time consuming. ${ }^{9,10}$ In recent years, electrochemical methods of detection have gathered enormous interest due to the simplicity, low cost, fast response time, low detection limit, high sensitivity and selectivity. ${ }^{11,12}$

Noble metal nanoparticles such as gold ( $\mathrm{Au})$, palladium (Pd), platinum (Pt) and silver (Ag) are broadly utilized as modified

\footnotetext{
${ }^{a}$ Department of Chemistry, Faculty of Science, University of Malaya, Kuala Lumpur, 50603, Malaysia.E-mail: sofiazira92@gmail.com; jeff@um.edu.my; Fax: +6037967 4193; Tel: +60379674082

${ }^{b}$ Institute of Nanotechnology and Catalysis (NanoCat), University of Malaya, Kuala Lumpur, 50603, Malaysia
}

$\dagger$ Electronic supplementary information (ESI) available. See DOI: 10.1039/c9ra11056a electrodes for electrochemical sensor applications. ${ }^{\mathbf{1 3 4}}$ The superior conductivity and electrocatalytic activity of Pt makes it an attractive material for sensor applications. ${ }^{15,16}$ Since Pt is overpriced due to the low natural abundance, ${ }^{17}$ attempts have been proposed to minimize the use of Pt without a compromise in the electrocatalytic activity. The first approach is the introduction of a secondary metal, such as $\mathrm{Ag}, \mathrm{Cu}, \mathrm{Fe}$ and $\mathrm{Ni}$, to form bimetallic nanoparticles. The bimetallic nanoparticles possess better electrochemical performance than the monometallic nanoparticles as a result of the synergistic effect between the two metals. ${ }^{18} \mathrm{Ag}$ nanoparticles are recommended for the alloying with $\mathrm{Pt}$ due to their biocompatibility, electrochemical stability, abundance, low cost and sustainable electrocatalytic activity. ${ }^{19}$ Therefore, the coupling of Ag with Pt could lead to an enhancement in the electrocatalytic activity of the bimetallic nanoparticles. The second approach is to select a suitable support material for the distribution of bimetallic nanoparticles. Graphene, a monolayer of $\mathrm{sp}^{2}$ hybridized carbon atoms in a hexagonal framework, ${ }^{20}$ meets these requirements as a support material due to its amazing physical and chemical properties. Graphene offers a high surface area, excellent electrical conductivity, good chemical and thermal stability, and low production cost. ${ }^{21}$ Thus, the combination of graphene and bimetallic nanoparticles could improve the electrocatalytic activity of the nanocomposite.

Thus, a nanocomposite of platinum-silver bimetallic nanoparticles supported on graphene $(\mathrm{Pt}-\mathrm{Ag} / \mathrm{Gr})$ was synthesized and characterized for morphological and structural properties. The electrochemical performance of the $\mathrm{Pt}-\mathrm{Ag} / \mathrm{Gr} / \mathrm{GCE}$ towards the detection of DA was analysed using voltammetric techniques. From these results, the bimetallic $\mathrm{Pt}-\mathrm{Ag} / \mathrm{Gr}$ 
nanocomposite showed enhancement in the electrocatalytic activity towards DA detection compared to single metallic $\mathrm{Pt} / \mathrm{Gr}$ and $\mathrm{Ag} / \mathrm{Gr}$ nanocomposites.

\section{Experimental methods}

\subsection{Materials}

Dipotassium hydrogen phosphate $\left(\mathrm{K}_{2} \mathrm{HPO}_{4}\right)$, potassium dihydrogen phosphate $\left(\mathrm{KH}_{2} \mathrm{PO}_{4}\right)$ and potassium tetrachloroplatinate $\left(\mathrm{K}_{2} \mathrm{PtCl}_{4}\right)$ were procured from Merck, while dopamine and silver nitrate $\left(\mathrm{AgNO}_{3}\right)$ were acquired from Sigma Aldrich. Dopamine hydrochloride (DA) injection was supplied by University Malaya Medical Centre (UMMC). All reagents and chemicals were of high purity and used as received. The supporting electrolyte was $0.1 \mathrm{~mol} \mathrm{~L}^{-1}$ phosphate buffer solution (PBS) at pH 6.5. Desired volumes of $\mathrm{K}_{2} \mathrm{HPO}_{4}$ and $\mathrm{KH}_{2} \mathrm{PO}_{4}$ were mixed with Milli-Q water for the preparation of PBS, while the DA stock solution $\left(0.01 \mathrm{~mol} \mathrm{~L}^{-1}\right)$ was prepared by dilution in PBS.

\subsection{Synthesis}

2.2.1. Synthesis of graphene. Graphene oxide (GO) was synthesized as the starting material for the preparation of graphene by a microwave-assisted method. ${ }^{22}$ First, graphite oxide was prepared from graphite following Hummers' method..$^{23}$ Five milligrams of the prepared graphite oxide powder was sonicated in $25 \mathrm{~mL}$ distilled water for $40 \mathrm{~min}$ in an ultrasonic bath to produce a GO suspension. Then, the suspension was heated by a microwave system for $5 \mathrm{~min}$. Subsequently, the graphene suspension was centrifuged before being placed in an oven at $60{ }^{\circ} \mathrm{C}$ for drying.

2.2.2. Synthesis of $\mathbf{P t}-\mathbf{A g} / \mathbf{G r}$ nanocomposite. The preparation of $\mathrm{Pt}-\mathrm{Ag} / \mathrm{Gr}$ was carried out by a chemical method as reported earlier. ${ }^{24}$ First, an equal amount of $0.05 \mathrm{~mol} \mathrm{~L}^{-1} \mathrm{AgNO}_{3}$ and $0.05 \mathrm{~mol} \mathrm{~L}^{-1} \mathrm{~K}_{2} \mathrm{PtCl}_{4}$ aqueous solutions were mixed with a $10 \mathrm{mg} \mathrm{mL}^{-1}$ graphene suspension. Next, $0.1 \mathrm{~mol} \mathrm{~L}^{-1}$ sodium hydroxide solution was used to adjust the $\mathrm{pH}$ of the mixture to 8.0. The resulting mixture was stirred and heated at $60{ }^{\circ} \mathrm{C}$ for $35 \mathrm{~min}$. This was followed by the addition of $0.01 \mathrm{~mL}$ hydrazine hydrate to the mixture while stirring and heating for an additional 30 min where the Ag and Pt nanoparticles were deposited onto the graphene surface to produce $\mathrm{Pt}-\mathrm{Ag} / \mathrm{Gr}$. Subsequently, the $\mathrm{Pt}-\mathrm{Ag} / \mathrm{Gr}$ suspension was centrifuged before drying in an oven at $60{ }^{\circ} \mathrm{C}$. The single metal $\mathrm{Ag} / \mathrm{Gr}$ and $\mathrm{Pt} / \mathrm{Gr}$ nanocomposites were also synthesized using the same procedure for comparisons.

2.2.3. Preparation of the $\mathbf{P t}-\mathbf{A g} / \mathrm{Gr}$ modified electrode. For the preparation of the modified electrode, a suspension was prepared by dispersing $1.0 \mathrm{mg} \mathrm{Pt}-\mathrm{Ag} / \mathrm{Gr}$ nanocomposite in $1.0 \mathrm{~mL}$ Milli-Q water. Then, $10 \mu \mathrm{L}$ of Nafion solution was added into the suspension, followed by ultrasonication for an hour to produce a homogenous dispersion. Next, $10 \mu \mathrm{L}$ of the suspension was pipetted using a micropipette and drop-casted onto the glassy carbon electrode (GCE) surface. Finally, the prepared electrode was dried overnight at room temperature. For comparisons, Ag/Gr/GCE, Pt/Gr/GCE and Gr/GCE were also prepared using the same procedures.

\subsection{Characterizations}

The crystal structures of the nanocomposite were evaluated by a PANalytical EMPYREAN X-ray diffractometer with $\mathrm{Cu} \mathrm{K} \alpha$ radiation $(\lambda=1.541 \AA)$. The surface morphology of the samples was analysed using JEOL JSM-7600F field emission scanning electron microscopy (FESEM), while the particle size was analysed by Hitachi HT-7700 transmission electron microscopy (TEM), attached with an EDAX TEAM energy dispersive X-ray (EDX) spectrometer. Raman spectroscopy was performed using a Renishaw inVia Raman microscope with a laser source of $532 \mathrm{~nm}$ excitation wavelength. The X-ray photoelectron spectroscopy (XPS) instrument was a ULVAC-PHI Quantera II Xray photoelectron spectrometer with an Al-K $\alpha$ X-ray source. Fourier transformed infrared (FTIR) spectra were obtained using a PerkinElmer Spectrum 400 FTIR spectrometer.

\subsection{Electrochemical study}

Electrochemical experiments such as cyclic voltammetry (CV), differential pulse voltammetry (DPV) and electrochemical impedance spectroscopy (EIS) were performed by an Autolab potentiostat/galvanostat model PGSTAT302 (EcoChemie, Netherlands). All electrochemical experiments were performed using a three-electrode system consisting of $\mathrm{Pt}-\mathrm{Ag} / \mathrm{Gr} / \mathrm{GCE}$ as the working electrode, saturated calomel electrode (SCE) as the reference electrode and a platinum wire as the counter electrode. All experiments were carried out at room temperature.

\section{Results and discussions}

\subsection{Characterization of $\mathbf{P t}-\mathbf{A g} / \mathbf{G r}$}

$\mathrm{XRD}$ was performed to identify the crystal structures of the $\mathrm{Ag} /$ $\mathrm{Gr}, \mathrm{Pt} / \mathrm{Gr}$ and $\mathrm{Pt}-\mathrm{Ag} / \mathrm{Gr}$ nanocomposites and to confirm the

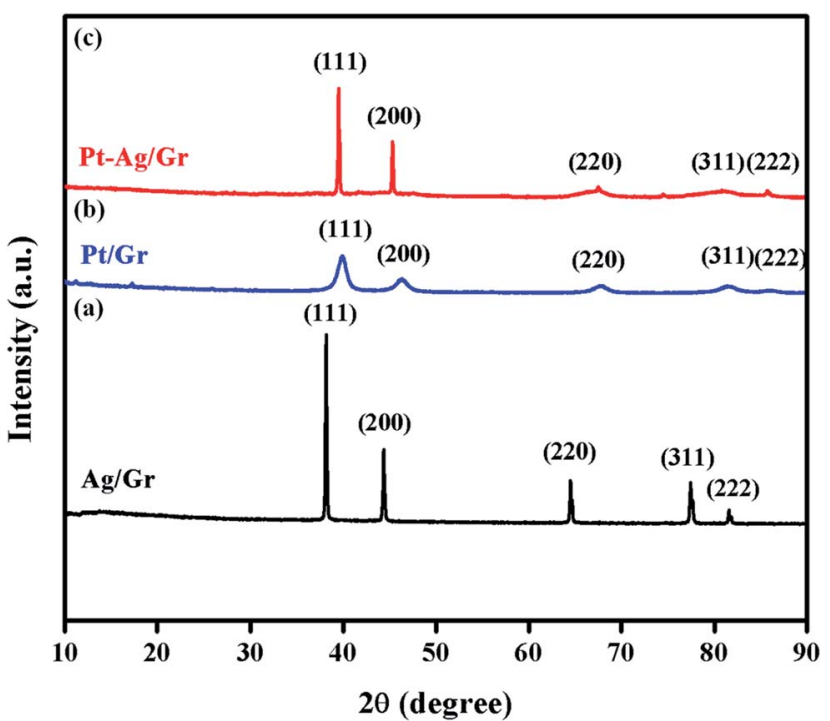

Fig. 1 The XRD pattern of (a) $\mathrm{Ag} / \mathrm{Gr}$ (b) $\mathrm{Pt} / \mathrm{Gr}$ and (c) $\mathrm{Pt}-\mathrm{Ag} / \mathrm{Gr}$ nanocomposites. 
formation of bimetallic Pt-Ag. Fig. 1a shows five diffraction peaks of $\mathrm{Ag}$ at $2 \theta$ values of $38.17^{\circ}, 44.35^{\circ}, 64.66^{\circ}, 77.66^{\circ}$ and $81.82^{\circ}$ which correspond to the (111), (200), (220), (311) and (222) crystal planes, respectively. Additionally, the XRD pattern of the Pt/Gr nanocomposite (Fig. 1b) demonstrates characteristic peaks at $2 \theta$ values of $39.87^{\circ}, 46.26^{\circ}, 67.68^{\circ}, 81.43^{\circ}$ and $86.12^{\circ}$, which are assigned to the (111), (200), (220), (311) and (222) crystal planes, respectively. According to the results, both $\mathrm{Ag}$ and Pt have a face-centred cubic (fcc) crystal structure (Ref. codes: 00-065-2871 and 01-001-1194). The diffraction peaks of $\mathrm{Pt}-\mathrm{Ag} / \mathrm{Gr}$ are present at $2 \theta$ values of $39.52^{\circ}, 45.32^{\circ}$, $66.28^{\circ}, 81.01^{\circ}$ and $85.70^{\circ}$ (Fig. 1c) and are situated between the values of pure Ag (Ref. code: 00-065-2871) and Pt (Ref. code: 01001-1194), which confirms the formation of bimetallic Pt-Ag. ${ }^{25}$ The mean particle size of $\mathrm{Pt}-\mathrm{Ag}$ is $6.3 \mathrm{~nm}$ from the Scherrer equation, while the $d$-spacing of $\mathrm{Pt}-\mathrm{Ag}$ is $0.228 \mathrm{~nm}$. The $d$ spacing value is between that of $\mathrm{Ag}(0.236 \mathrm{~nm})$ and Pt $(0.226$ $\mathrm{nm})$.

The morphological features of the as-synthesized $\mathrm{Pt}-\mathrm{Ag} / \mathrm{Gr}$ nanocomposite were analysed by FESEM and TEM imaging. The FESEM image in Fig. 2a illustrates the deposition of Pt-Ag nanoparticles on the wrinkled sheet structures of graphene. TEM analysis was performed to further validate the FESEM characterization. Fig. 2b presents the TEM image of the $\mathrm{Pt}-\mathrm{Ag} /$ Gr nanocomposite. The TEM image shows that the graphene sheets are decorated with small spherical Ag and Pt nanoparticles. The mean nanoparticle size ( $n=55$, Fig. $2 \mathrm{c})$ of Pt-Ag is approximately $6.50 \mathrm{~nm}$, in the range of $4.0-14.0 \mathrm{~nm}$. The small size of the nanoparticles and narrow particle size distribution suggest that the graphene support plays an essential role in the nucleation of the nanoparticles. The presence of carbon, oxygen, platinum and silver in the $\mathrm{Pt}-\mathrm{Ag} / \mathrm{Gr}$ nanocomposite was detected by EDX as shown in Fig. S1a. $\uparrow$ EDX elemental mapping was used to investigate the distribution of $\mathrm{Pt}$ and $\mathrm{Ag}$ nanoparticles on the graphene surface. Fig. S1b $\dagger$ illustrates the elemental mapping images of carbon, oxygen, silver and platinum. The EDX elemental mapping (Fig. S1b $\dagger$ ) shows the presence of bimetallic Pt-Ag nanoparticles on the graphene surface. Fig. 2d presents a higher magnification TEM image of the Pt-Ag lattice fringes. The lattice fringe spacing is approximately $0.230 \mathrm{~nm}$, attributed to the (111) crystal plane of facecentred cubic $\mathrm{Pt}-\mathrm{Ag}$. The result is similar to the $d$-spacing value obtained from the XRD analysis. Fig. 2e presents the selected area electron diffraction (SAED) image with a definite ring, which reveals the presence of a crystalline material. ${ }^{21}$

The structural characteristics of carbon compounds especially graphene based materials can be investigated by Raman spectroscopy. The Raman spectra of GO, graphene and the Pt$\mathrm{Ag} / \mathrm{Gr}$ nanocomposite are shown in Fig. 3. Two distinctive bands at approximately $1360 \mathrm{~cm}^{-1}$ and $1580 \mathrm{~cm}^{-1}$ were identified in all the samples, which are attributed to the D and $\mathrm{G}$ bands, respectively. The presence of the $\mathrm{D}$ band is related to the defects and disorder in the graphene structure, ${ }^{\mathbf{2 6}}$ whereas the $\mathrm{G}$ band originates from the $\mathrm{E}_{2 \mathrm{~g}}$ vibrations of the $\mathrm{sp}^{2}$ carbon atoms. ${ }^{27,28}$ The intensity ratio of the $\mathrm{D}$ band and $\mathrm{G}$ band $\left(I_{\mathrm{D}} / I_{\mathrm{G}}\right)$ represents the amount of disorder in carbon compounds. ${ }^{29}$ The $I_{\mathrm{D}} / I_{\mathrm{G}}$ of GO and graphene is 0.808 and 0.869 , respectively, which suggests an increase in the defects in graphene synthesized from the reduction of GO with microwave irradiation. Furthermore, the $I_{\mathrm{D}} / I_{\mathrm{G}}$ ratio of the $\mathrm{Pt}-\mathrm{Ag} / \mathrm{Gr}$ nanocomposite (0.950) shows an increase in the defect density which confirms structural distortion of graphene due to the incorporation of $\mathrm{Pt}-\mathrm{Ag}$ nanoparticles.
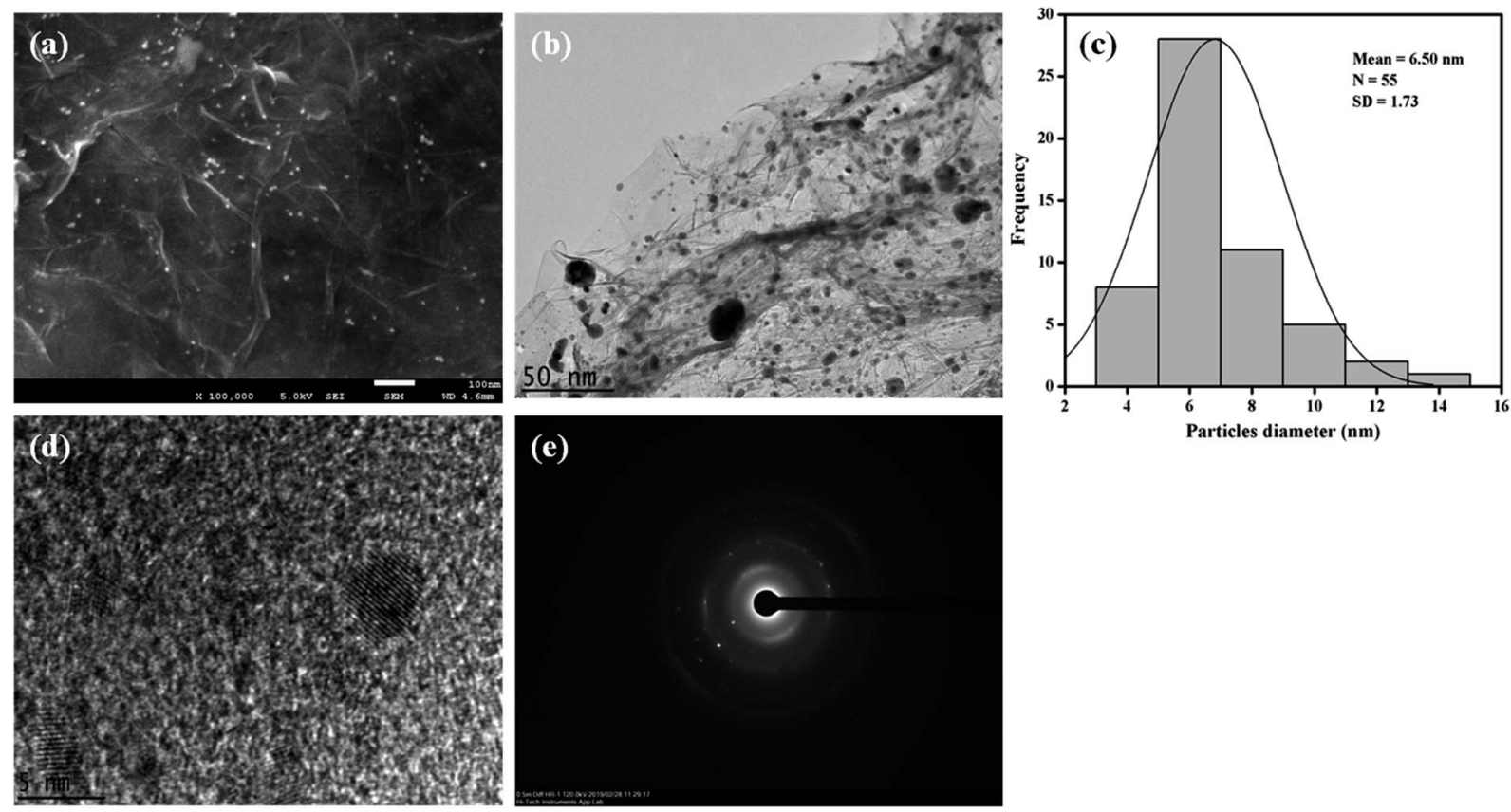

Fig. 2 (a) FESEM image of Pt-Ag/Gr. (b) TEM image of Pt-Ag/Gr. (c) The particle size distribution histogram of Pt-Ag. (d) HRTEM image of Pt-Ag/ Gr. (e) SAED pattern of Pt-Ag. 


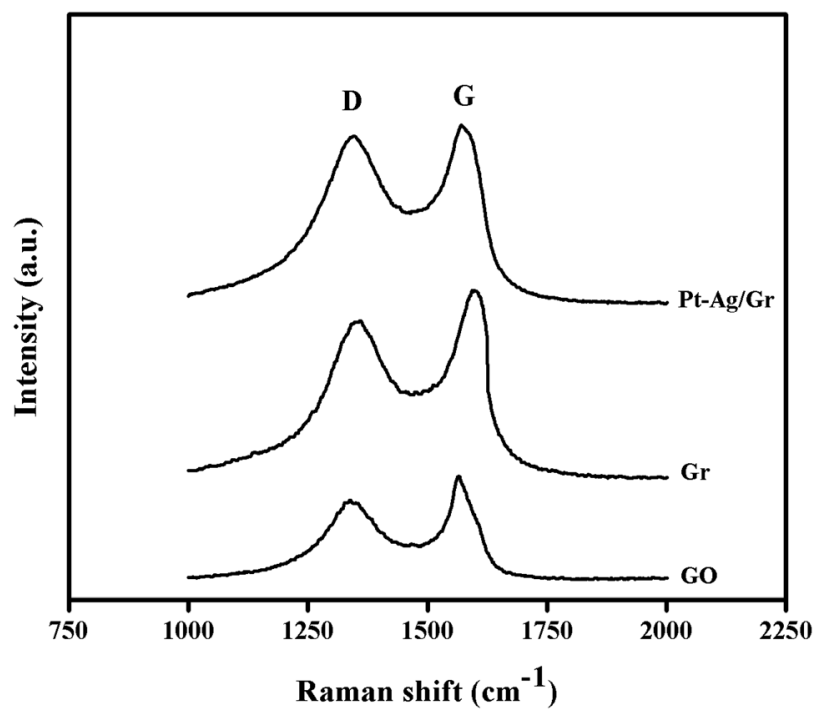

Fig. 3 Raman spectra of GO, Gr and Pt-Ag/Gr.

The chemical state and elemental composition of the prepared $\mathrm{Pt}-\mathrm{Ag} / \mathrm{Gr}$ nanocomposite were characterized by XPS. Fig. S2(a-d) $\dagger$ shows the survey scan spectrum of $\mathrm{Pt}-\mathrm{Ag} / \mathrm{Gr}$ with the deconvolution of the $\mathrm{C} 1 \mathrm{~s}, \mathrm{Ag} 3 \mathrm{~d}$ and $\mathrm{Pt} 4 \mathrm{f}$ core level spectra. Three predominant peaks are present in the survey scan spectrum of $\mathrm{Pt}-\mathrm{Ag} / \mathrm{Gr}$ (Fig. S2a $\dagger$ ) which are attributed to the $\mathrm{C} 1 \mathrm{~s}, \mathrm{Ag}$ $3 \mathrm{~d}$ and $\mathrm{Pt} 4 \mathrm{f}$ levels. The $\mathrm{C} 1 \mathrm{~s}$ core level spectra of $\mathrm{Pt}-\mathrm{Ag} / \mathrm{Gr}$ in Fig. S2b $\dagger$ shows three distinct peaks located at binding energies of $284.63 \mathrm{eV}, 285.68 \mathrm{eV}$ and $287.31 \mathrm{eV}$, which are ascribed to the $\mathrm{C}=\mathrm{C}, \mathrm{C}-\mathrm{O}$ and $\mathrm{C}=\mathrm{O}$ groups, respectively. As shown in Fig. S2c,$\dagger$ the deconvolution of the $\mathrm{Ag} 3 \mathrm{~d}$ spectrum shows a doublet of $\mathrm{Ag}$ $3 \mathrm{~d}_{5 / 2}$ and $\mathrm{Ag} 3 \mathrm{~d}_{3 / 2}$ due to spin-orbit splitting which is located at $368.24 \mathrm{eV}$ and $374.25 \mathrm{eV}$, respectively. Furthermore, the Pt $4 \mathrm{f}_{7 / 2}$ and $\mathrm{Pt} 4 \mathrm{f}_{5 / 2}$ peaks can be observed in the high-resolution Pt $4 \mathrm{f}$ spectra of the $\mathrm{Pt}-\mathrm{Ag} / \mathrm{Gr}$ nanocomposite, as presented in Fig. S2d. $\dagger$ Each peak of $\mathrm{Pt} 4 \mathrm{f}_{7 / 2}$ and $\mathrm{Pt} 4 \mathrm{f}_{5 / 2}$ can be deconvoluted into three different oxidation states, namely the $\mathrm{Pt}^{0}, \mathrm{Pt}^{2+}$ and $\mathrm{Pt}^{4+} \cdot \mathrm{Pt}^{0} 4 \mathrm{f}_{7 / 2}$ and $\mathrm{Pt}^{0} 4 \mathrm{f}_{5 / 2}$ and are centred at binding energies of $71.14 \mathrm{eV}$ and $74.69 \mathrm{eV}$, respectively. The two distinct peaks at $72.56 \mathrm{eV}$ and $76.37 \mathrm{eV}$ are characteristic of the $\mathrm{Pt}^{2+} 4 \mathrm{f}_{7 / 2}$ and $\mathrm{Pt}^{2+} 4 \mathrm{f}_{5 / 2}$ levels, respectively, whereas the peaks at $74.21 \mathrm{eV}$ and $78.24 \mathrm{eV}$ are assigned to the $\mathrm{Pt}^{4+} 4 \mathrm{f}_{7 / 2}$ and $\mathrm{Pt}^{4+} 4 \mathrm{f}_{5 / 2}$, respectively. The XPS results confirmed that both Ag NPs and Pt NPs are present on the graphene surface, thus the $\mathrm{Pt}-\mathrm{Ag} / \mathrm{Gr}$ nanocomposite was successfully synthesized.

Fig. 4 presents the FTIR spectra of GO, Gr and $\mathrm{Pt}-\mathrm{Ag} / \mathrm{Gr}$ in the range of $500-4000 \mathrm{~cm}^{-1}$ to investigate the functional groups. ${ }^{30} \mathrm{~A}$ broad band at $3270 \mathrm{~cm}^{-1}$ in the FTIR spectrum of GO (Fig. 4a) is assigned to the $\mathrm{O}-\mathrm{H}$ stretching vibration of the GO sheets. ${ }^{31}$ The characteristic peaks at 2929, 1723, 1626, 1366 and $998 \mathrm{~cm}^{-1}$ correspond to the stretching vibrations of $\mathrm{C}-\mathrm{H}, \mathrm{C}=\mathrm{O}, \mathrm{C}=\mathrm{C}$, $\mathrm{C}-\mathrm{O}-\mathrm{H}$ and $\mathrm{C}-\mathrm{O}$, respectively. ${ }^{19,25}$ These results confirm that GO consists of oxygen functional groups. ${ }^{\mathbf{6}, 31}$ The peak intensities of the oxygen functional groups were diminished in Gr (Fig. 4b). In addition, some of the peaks were absent in $\mathrm{Pt}-\mathrm{Ag} / \mathrm{Gr}$ (Fig. 4c) which is likely due to the presence of Pt-Ag nanoparticles on the

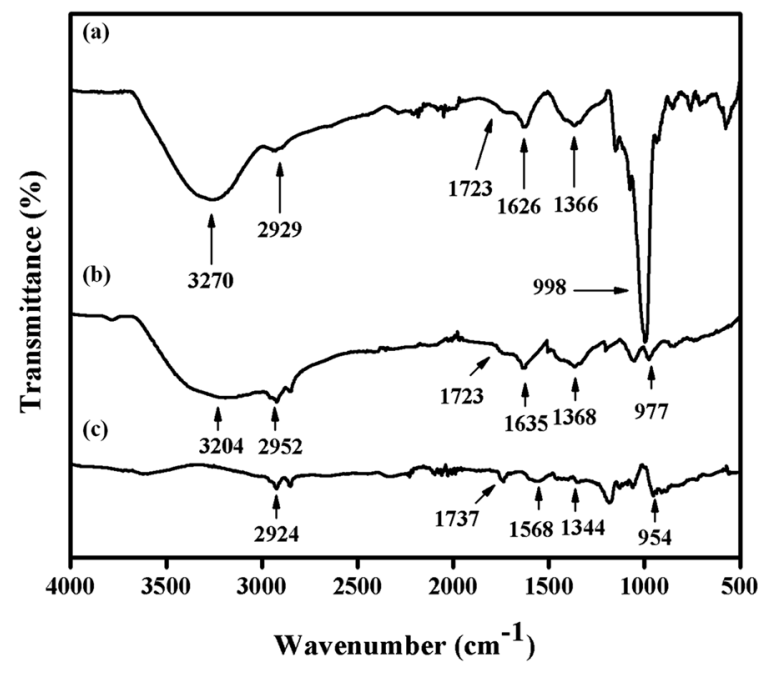

Fig. 4 FTIR spectra of (a) GO, (b) Gr and (c) Pt-Ag/Gr.

graphene surface. ${ }^{5,32}$ Thus, these results confirm that the oxygen functional groups were reduced in $\mathrm{Pt}-\mathrm{Ag} / \mathrm{Gr}$.

\subsection{Electrochemical impedance spectroscopy (EIS) studies}

The interfacial electron transfer resistance $\left(R_{\mathrm{et}}\right)$ of the modified electrodes was studied by EIS. ${ }^{33}$ Fig. S3† presents the Nyquist plots of the modified electrodes in a $5.0 \mathrm{mM} \mathrm{Fe}\left[(\mathrm{CN})_{6}\right]^{3-/ 4-}$ redox couple in $0.1 \mathrm{M} \mathrm{KCl}$ between $100 \mathrm{kHz}$ and $100 \mathrm{mHz}$. The Nyquist plots shows a semicircle at low frequency where the diameter corresponds to the interfacial electron transfer resistance $\left(R_{\mathrm{et}}\right)$ of the modified electrodes. The low-frequency region shows a straight line $45^{\circ}$ (Warburg element) to the horizontal axis, is due to a diffusion-limited process. The bare GCE shows the largest semicircle diameter which represents the largest electron transfer resistance. The semicircle diameter is considerably decreased when graphene is immobilized on the surface of the GCE. This is due to the high electrical conductivity of graphene, which decreases the impedance of the GCE. In addition, the semicircle diameter decreases progressively from $\mathrm{Gr} / \mathrm{GCE}$ to $\mathrm{Ag} / \mathrm{Gr} / \mathrm{GCE}, \mathrm{Pt} / \mathrm{Gr} / \mathrm{GCE}$ and $\mathrm{Pt}-\mathrm{Ag} / \mathrm{Gr} / \mathrm{GCE}$ with the introduction of Ag NPs and Pt NPs on the graphene surface. This demonstrates the decreasing trend of the electron transfer resistance due to the deposition of conductive metal nanoparticles. The $R_{\text {et }}$ values of GCE, Gr/GCE, Ag/Gr/GCE, Pt/Gr/GCE and $\mathrm{Pt}-\mathrm{Ag} / \mathrm{Gr} / \mathrm{GCE}$ were obtained by fitting and simulation of the experimental impedance data with a suitable equivalent circuit. The $R_{\text {et }}$ values decrease in the order of GCE $(824 \Omega)>\mathrm{Gr} /$ $\operatorname{GCE}(533 \Omega)>\mathrm{Ag} / \mathrm{Gr} / \mathrm{GCE}(130 \Omega)>\mathrm{Pt} / \mathrm{Gr} / \mathrm{GCE}(116 \Omega)>\mathrm{Pt}-\mathrm{Ag} /$ Gr/GCE (69 $\Omega$ ). The $R_{\text {et }}$ values of $\mathrm{Ag} / \mathrm{Gr} / \mathrm{GCE}$ and $\mathrm{Pt} / \mathrm{Gr} / \mathrm{GCE}$ are lower than those of Gr/GCE, implying that the presence of conductive metal nanoparticles facilitates the electron transfer process. Furthermore, $\mathrm{Pt}-\mathrm{Ag} / \mathrm{Gr} / \mathrm{GCE}$ shows the lowest $R_{\mathrm{et}}$ values due to the synergistic effect of the bimetallic $\mathrm{Pt}-\mathrm{Ag}$ nanoparticles and graphene which enhances the electrical conductivity and decreases the interfacial resistance. For this reason, $\mathrm{Pt}-\mathrm{Ag} / \mathrm{Gr} / \mathrm{GCE}$ was utilized for the development of the electrochemical sensor. 


\subsection{Optimization of experimental conditions}

3.3.1. Effect of $\mathbf{p H}$. The $\mathrm{pH}$ of the electrolyte significantly affects the electrochemical performance of DA at Pt-Ag/Gr/GCE. The cyclic voltammetry of DA were performed at different $\mathrm{pH}$ in $0.1 \mathrm{~mol} \mathrm{~L}^{-1}$ PBS between $\mathrm{pH} 5.5$ and 7.5 in the presence of 25 $\mu \mathrm{M}$ DA. From Fig. S4a, $\uparrow$ it can be seen that the peak potential for DA oxidation becomes less positive as the $\mathrm{pH}$ increases from 5.5 to 7.5 , which confirms the involvement of protons in the electrochemical oxidation of DA. ${ }^{34}$ Furthermore, good linearity was achieved between the oxidation peak potentials and the $\mathrm{pH}$ values, as presented in Fig. $\mathrm{S} 4 \mathrm{~b}, \dagger$ with a linear regression equation of $E_{\mathrm{pa}}(\mathrm{V})=-0.064 \mathrm{pH}+0.681$. From this equation, the obtained slope of $0.064 \mathrm{~V} \mathrm{pH}^{-1}$ is close to the theoretical value from the Nernst equation $\left(0.059 \mathrm{~V} \mathrm{pH}^{-1}\right)$ at standard temperature. ${ }^{35}$ This confirms that the electrochemical oxidation of DA involves an equal number of protons and electrons. ${ }^{36}$ The plot in Fig. S4c $\dagger$ illustrates that the DA oxidation peak current increases with the $\mathrm{pH}$ value from 5.5 to 6.5 but declines beyond $\mathrm{pH}$ 6.5. Therefore, $\mathrm{pH} 6.5$ was chosen as the ideal $\mathrm{pH}$ for the electrochemical detection of DA in the subsequent experiments due to the highest DA oxidation peak current.

3.3.2. Effect of scan rate. The influence of the scan rate on the redox peak currents of DA oxidation with Pt-Ag/Gr/GCE was studied by cyclic voltammetry. The cyclic voltammetric measurements were performed with the $\mathrm{Pt}-\mathrm{Ag} / \mathrm{Gr} / \mathrm{GCE}$ at several scan rates from 5 to $200 \mathrm{mV} \mathrm{s}^{-1}$ in $0.1 \mathrm{~mol} \mathrm{~L}^{-1}$ PBS containing $25 \mu \mathrm{M}$ DA as shown in Fig. S5a. $\uparrow$ The redox peak current of DA increases with the scan rate, and a linear correlation between the parameters was observed. The regression equations shown in Fig. S5b $\dagger$ can be expressed as $I_{\mathrm{pa}}(\mu \mathrm{A})=0.154 \nu+0.676\left(R^{2}=0.988\right)$ and $I_{\mathrm{pc}}(\mu \mathrm{A})=$ $-0.191 \nu-7.55\left(R^{2}=0.971\right)$, which confirm that the electrochemical process is a surface adsorption controlled reaction. ${ }^{37}$ Furthermore, when the scan rate increases, the oxidation and reduction peak potentials are slightly shifted to positive and negative regions, respectively, which is associated with a slower electron transfer process at faster scan rates. Fig. S5c $\dagger$ demonstrates the linear relationship of the redox peak potential and the logarithm of the scan rate in the range of 5 to $75 \mathrm{mV} \mathrm{s}^{-1}$, with regression equations of $E_{\mathrm{pa}}=0.0786 \log \nu+0.184\left(R^{2}=0.962\right)$ and $E_{\mathrm{pc}}=-0.0564 \log \nu+0.104\left(R^{2}=0.949\right)$. Based on the Laviron equation, the slopes of the two lines are 2.303RT/(1- $\alpha) n F$ and $-2.303 R T / \alpha n F$. From the calculation, the electron transfer coefficient $(\alpha)$ and the number of electrons involved in the reaction are 0.58 and $1.8 \approx 2$, respectively. Therefore, two electrons are required in the electrochemical redox reaction of DA.

\subsection{Electrochemical behaviour of dopamine on $\mathrm{Pt}-\mathrm{Ag} / \mathrm{Gr} /$ GCE}

The electrochemical performance of DA at the GCE, Gr/GCE, Ag/ Gr/GCE, Pt/Gr/GCE and Pt-Ag/Gr/GCE was investigated by cyclic voltammetry in $0.1 \mathrm{~mol} \mathrm{~L}^{-1} \mathrm{PBS}$ ( $\mathrm{pH} 6.5$ ) containing $25 \mu \mathrm{M}$ DA at $20 \mathrm{mV} \mathrm{s}^{-1}$. In Fig. 5, only the oxidation peak current of DA was observed at the GCE due to the sluggish electrochemical reaction of DA on the GCE surface. In contrast, the Gr/GCE exhibited a pair of small redox peak currents. The DA oxidation peak current of the Gr/GCE increases slightly compared to the GCE

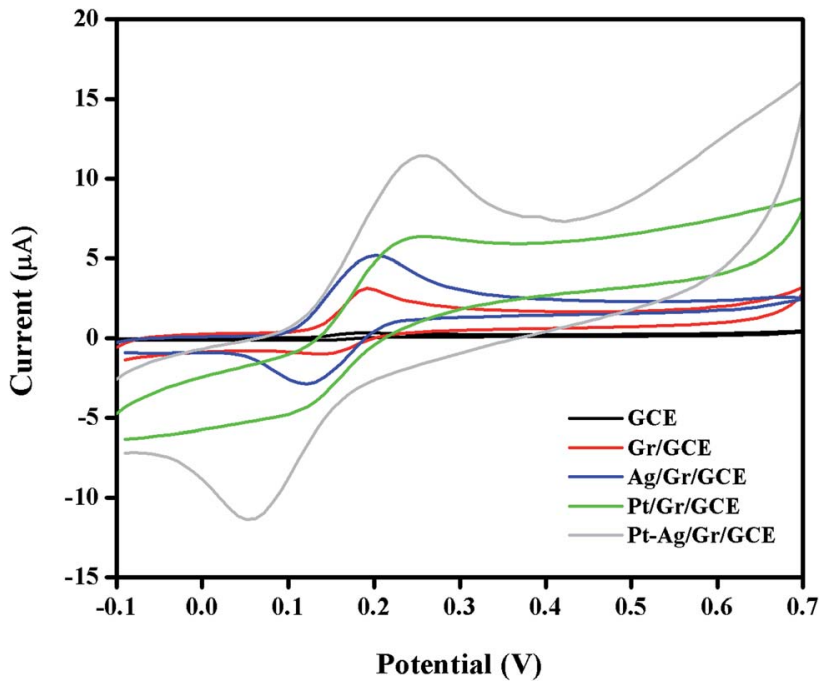

Fig. 5 Cyclic voltammograms of $25 \mu \mathrm{M} \mathrm{DA}$ in $0.1 \mathrm{~mol} \mathrm{~L}^{-1} \mathrm{PBS}(\mathrm{pH} 6.5)$ at a scan rate of $20 \mathrm{mV} \mathrm{s}^{-1}$ on bare GCE, Gr/GCE, Ag/Gr/GCE, Pt/Gr/ GCE and Pt-Ag/Gr/GCE.

due to the high surface area and high electron conductivity of graphene, which provides more active sites for the electrochemical reaction. ${ }^{38}$ Moreover, the presence of metal nanoparticles on the graphene surface facilitates the electron transfer reaction during the electrochemical detection of DA. The cyclic voltammograms of Ag/Gr/GCE, Pt/Gr/GCE and Pt-Ag/ Gr/GCE show clear DA oxidation peak currents. The Pt-Ag/Gr/ GCE shows the highest DA oxidation peak current compared to the $\mathrm{Ag} / \mathrm{Gr} / \mathrm{GCE}$ and $\mathrm{Pt} / \mathrm{Gr} / \mathrm{GCE}$, confirming the outstanding electrocatalytic activity of $\mathrm{Pt}-\mathrm{Ag} / \mathrm{Gr}$ towards DA oxidation. The enhancement in the peak current is ascribed to the synergistic effects between the Pt-Ag bimetallic nanoparticles and graphene. The unique combination of both $\mathrm{Pt}$ and $\mathrm{Ag}$ provides excellent electrocatalytic performance and superior electronic conductivity compared to single metal $\mathrm{Pt}$ and $\mathrm{Ag}$ nanoparticles. Furthermore, the incorporation of $\mathrm{Pt}$ and $\mathrm{Ag}$ bimetallic nanoparticles on the high surface area of graphene contributes to more electroactive sites for efficient electron transfer between the modified electrode and analyte. ${ }^{39}$ Thus the $\mathrm{Pt}-\mathrm{Ag} / \mathrm{Gr}$ nanocomposite modified electrode is recommended for the electrochemical detection of DA due to the superior conductivity, excellent electrocatalytic activity and high surface area.

\subsection{Analytical application of the sensor}

The differential pulse voltammetry (DPV) method was selected for the quantitative analysis of DA on $\mathrm{Pt}-\mathrm{Ag} / \mathrm{Gr} / \mathrm{GCE}$ due to the better sensitivity and lower detection limit than $\mathrm{CV} .^{40}$ The DPV responses of the sensor with various concentrations of DA in $0.1 \mathrm{~mol} \mathrm{~L}^{-1}$ PBS are presented in Fig. 6a. Based on the DPV results, the DA oxidation peak current increases with the DA concentration, from 0.1 to $60 \mu \mathrm{M}$. Fig. $6 \mathrm{~b}$ demonstrates the excellent linearity of the calibration curve between the oxidation peak current and DA concentration, with a linear equation $I_{\mathrm{pa}}$ $(\mu \mathrm{A})=0.309 C(\mu \mathrm{M})-0.0699\left(R^{2}=0.997\right)$. The detection limit of 

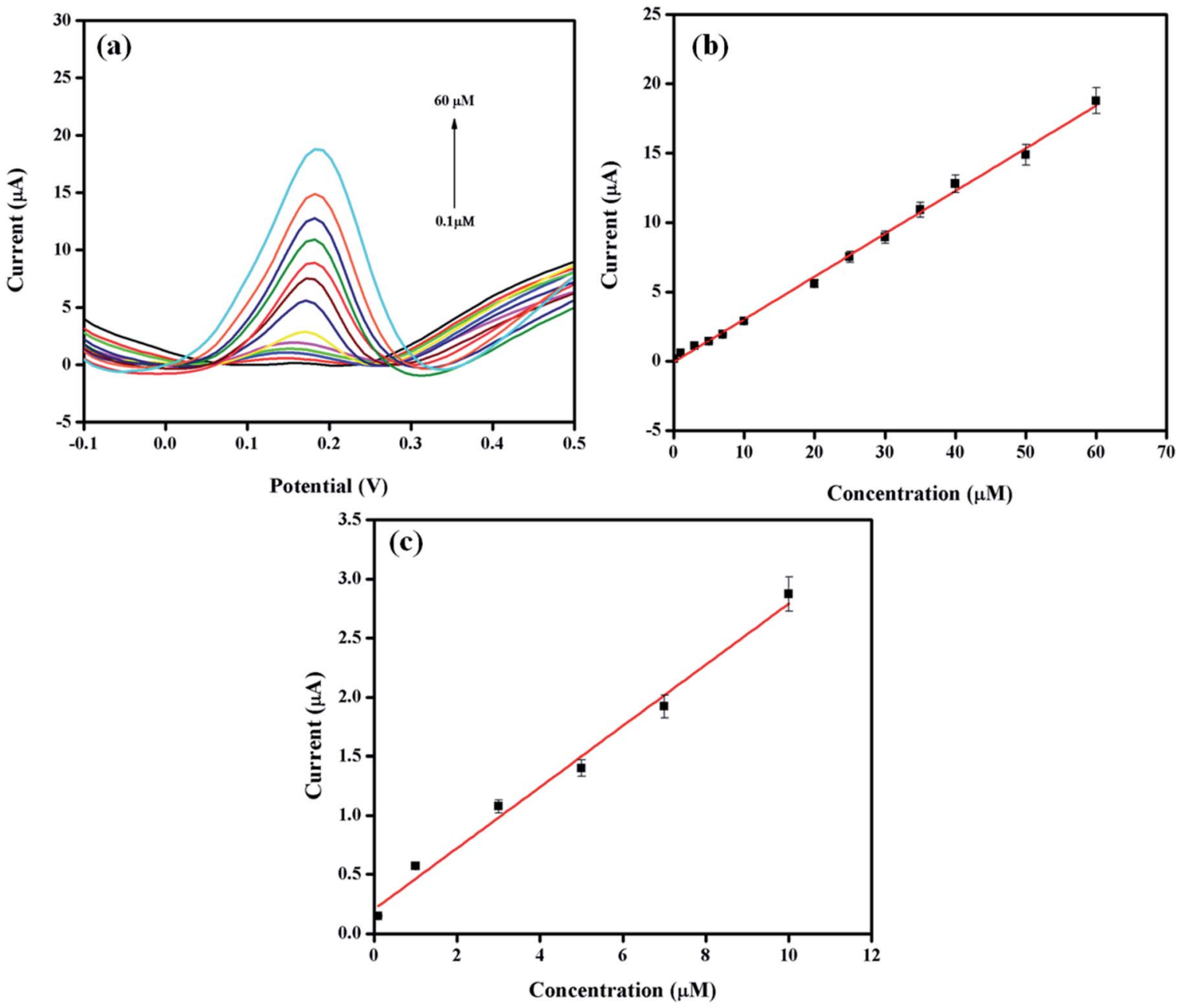

Fig. 6 (a) Differential pulse voltammograms with various DA concentrations $(0.1,1,3,5,710,20,25,30,35,40,50,60 \mu \mathrm{M})$ at $\mathrm{Pt}-\mathrm{Ag} / \mathrm{Gr} / \mathrm{GCE}$ in $0.1 \mathrm{~mol} \mathrm{~L}^{-1} \mathrm{PBS}$ at pH 6.5. (b) Relationship between peak current and DA concentration. (c) The calibration curve in low concentration range.

DA is $0.012 \mu \mathrm{M}$ based on a signal-to-noise ratio $(\mathrm{S} / \mathrm{N})$ of 3 . The performance of $\mathrm{Pt}-\mathrm{Ag} / \mathrm{Gr}$ is compared with other modified electrodes for DA detection, as summarized in Table 1 . The

Table 1 Comparison of analytical performance on different electrode materials for DA detection by DPV method

\begin{tabular}{llll}
\hline $\begin{array}{l}\text { Electrode } \\
\text { materials }\end{array}$ & $\begin{array}{l}\text { Linear } \\
\text { range }(\mu \mathrm{M})\end{array}$ & $\begin{array}{l}\text { Detection limit } \\
(\mu \mathrm{M})\end{array}$ & References \\
\hline $\mathrm{Au}-\mathrm{Cu}_{2} \mathrm{O} / \mathrm{rGO}$ & $10-90$ & 3.9 & 38 \\
$\mathrm{~N}-\mathrm{rGO}$ & $3-70$ & 1.5 & 7 \\
$\mathrm{Au} / \mathrm{PDDA} / \mathrm{GNS}$ & $2-28$ & 1.0 & 41 \\
$\mathrm{PdAu} / \mathrm{rGO}$ & $1.25-73.75$ & 0.75 & 42 \\
$\mathrm{AG}-\mathrm{NA}$ & $0.5-35$ & 0.33 & 2 \\
$\mathrm{GNP} / \mathrm{FTO}$ & $30-100$ & 0.22 & 35 \\
$\mathrm{PGE}$ & $0.2-8$ & 0.20 & 43 \\
$\mathrm{NGF}$ & $0.1-80$ & 0.030 & 44 \\
$\mathrm{PANI}-\mathrm{rGO}$ & $0.05-60$ & 0.024 & 6 \\
$\beta-\mathrm{CD} / \mathrm{rGO}$ & $0.05-50$ & 0.017 & 36 \\
$\mathrm{PA} / \mathrm{GO}$ & $0.05-10$ & 0.016 & 45 \\
$\mathrm{Pt}-\mathrm{Ag} / \mathrm{Gr}$ & $0.1-60$ & 0.012 & {$[$ This work] }
\end{tabular}

detection limit of $\mathrm{Pt}-\mathrm{Ag} / \mathrm{Gr} / \mathrm{GCE}$ is comparable to $\beta-\mathrm{CD} / \mathrm{rGO}$ and $\mathrm{PA} / \mathrm{GO}$ and is even better than $\mathrm{Au}-\mathrm{Cu}_{2} \mathrm{O} / \mathrm{rGO}, \mathrm{N}-\mathrm{rGO}, \mathrm{Au} / \mathrm{PDDA} /$ GNS, PdAu/rGO, AG-NA, GNP/FTO, PGE, NGF and PANI/rGO. No perceptible differences were observed between the linear ranges of the present sensor with N-rGO, PdAu/rGO, NGF, PANI/rGO and $\beta-\mathrm{CD} / \mathrm{rGO}$. Thus, it can be concluded that the high sensitivity of $\mathrm{Pt}-\mathrm{Ag} / \mathrm{Gr} / \mathrm{GCE}$ towards the electrochemical determination of DA is due to the synergistic effects of bimetallic Pt-Ag NPs and graphene.

\subsection{Stability and reproducibility of $\mathrm{Pt}-\mathrm{Ag} / \mathrm{Gr} / \mathrm{GCE}$}

The stability and reproducibility of the $\mathrm{Pt}-\mathrm{Ag} / \mathrm{Gr}$ were tested to determine the efficiency of the sensor. For stability, the DA oxidation peak current was measured after storage of $\mathrm{Pt}-\mathrm{Ag} / \mathrm{Gr} /$ GCE in PBS for two weeks in a refrigerator. The electrochemical response of DA was reduced to approximately $11.8 \%$ of the initial current response value. Five different $\mathrm{Pt}-\mathrm{Ag} / \mathrm{Gr} / \mathrm{GCEs}$ were prepared individually by the same method to investigate the reproducibility of the sensor towards DA detection. From the analysis, the relative standard deviation (RSD) of the DA oxidation peak current is estimated as 3.01\%. These results 
Table 2 DA determination in dopamine hydrochloride injection using $\mathrm{Pt}-\mathrm{Ag} / \mathrm{Gr} / \mathrm{GCE}$

\begin{tabular}{llll}
\hline Content $(\mu \mathrm{M})$ & Detected $(\mu \mathrm{M})$ & Recovery $(\%)$ & RSD $(\%)$ \\
\hline 3.00 & 2.74 & 91.4 & 2.94 \\
8.00 & 7.92 & 99.0 & 1.25 \\
10.0 & 9.56 & 95.6 & 0.58 \\
12.0 & 11.9 & 98.8 & 1.76
\end{tabular}

suggest an acceptable storage stability and reproducibility of the prepared electrode.

\subsection{Selectivity of $\mathbf{P t}-\mathbf{A g} / \mathbf{G r} / \mathbf{G C E}$}

The selectivity of a sensor towards a target analyte is one of the most essential characteristics of a high-performance sensor due to the presence of interfering compounds that also oxidize in the same potential range. ${ }^{8}$ The selective determination of DA in the presence of biological compounds such as ascorbic acid (AA), $p$-aminophenol (PAP), acetaminophen (AC) and uric acid (UA) was performed using DPV by varying the concentration of DA in a solution with a constant concentration of the interfering compounds. As shown in Fig. S6a, $\dagger$ the peak currents of AA, PAP, DA, AC and UA are separated from each other. Moreover, a linear relationship is observed between the DA peak current and the concentration, with the linear equation of $I_{\mathrm{pa}}(\mu \mathrm{A})=$ $0.292 C(\mu \mathrm{M})+14.59$ as illustrated in Fig. S6b. $\uparrow$ The sensitivity of the $\mathrm{Pt}-\mathrm{Ag} / \mathrm{Gr}$ modified electrode in the presence of interferences (0.292 $\mathrm{A} \mathrm{M}^{-1}$ ) decreases only approximately $5.5 \%$ from the sensitivity of $\mathrm{Pt}-\mathrm{Ag} / \mathrm{Gr} / \mathrm{GCE}$ in the absence of interference (0.309 $\mathrm{A} \mathrm{M}^{-1}$ ). This implies that the presence of AA, PAP, AC and UA in the same mixture has a negligible effect on the detection of DA. Thus, Pt-Ag/Gr/GCE possesses acceptable selectivity for the detection of DA in the presence of common interferences.

\subsection{Real sample analysis}

Dopamine hydrochloride injection $\left(40 \mathrm{mg} \mathrm{mL}^{-1}\right)$ was used for the determination of $\mathrm{DA}$ at the $\mathrm{Pt}-\mathrm{Ag} / \mathrm{Gr}$ modified electrode in real sample analysis. The dopamine hydrochloride injection was diluted with $0.1 \mathrm{~mol} \mathrm{~L}^{-1} \mathrm{PBS}(\mathrm{pH}$ 6.5) for a suitable concentration range in the determination of DA. Several concentrations of dopamine hydrochloride injection were prepared, and the current response was examined using the DPV method under the same experimental conditions as before. From the results summarized in Table 2, the obtained recoveries are between $91.4-99.0 \%$. Therefore, the fabricated sensor shows acceptable reliability for DA determination in real samples.

\section{Conclusions}

In summary, a GCE surface was modified with $\mathrm{Pt}-\mathrm{Ag} / \mathrm{Gr}$ nanocomposite for the electrochemical determination of DA. From the results, the combination of graphene and $\mathrm{Pt}-\mathrm{Ag}$ nanoparticles greatly improved the oxidation peak current of DA. The outstanding electrocatalytic activity of $\mathrm{Pt}-\mathrm{Ag} / \mathrm{Gr}$ towards DA was due to the synergistic effects of the high surface area of graphene and superior electronic conductivity of the Pt-Ag nanoparticles. The obtained Pt-Ag/Gr modified GCE had acceptable sensitivity, selectivity, stability, reproducibility and a low detection limit. With this remarkable performance, the prepared sensor was tested for the determination of DA in real samples with satisfying results.

\section{Conflicts of interest}

There are no conflicts of interest to declare.

\section{Acknowledgements}

The authors would like to thank the University of Malaya under the Postgraduate Research Grant (PPP) PG093-2015B for the funding of this research project.

\section{References}

1 V. K. Yeragani, M. Tancer, P. Chokka and G. B. Baker, Arvid Carlsson, and the story of dopamine, Indian J. Psychiatr., 2010, 52, 87-88.

2 D. Kim, S. Lee and Y. Piao, Electrochemical determination of dopamine and acetaminophen using activated grapheneNafion modified glassy carbon electrode, J. Electroanal. Chem., 2017, 794, 221-228.

3 H. W. Yu, J. H. Jiang, Z. Zhang, G. C. Wan, Z. Y. Liu, D. Chang and H. Z. Pan, Preparation of quantum dots CdTe decorated graphene composite for sensitive detection of uric acid and dopamine, Anal. Biochem., 2017, 519, 92-99.

4 J. Fang, Z. Xie, G. Wallace and X. Wang, Co-deposition of carbon dots and reduced graphene oxide nanosheets on carbon-fiber microelectrode surface for selective detection of dopamine, Appl. Surf. Sci., 2017, 412, 131-137.

5 V. Sharma, A. Sundaramurthya, A. Tiwari and A. K. Sundramoorthy, Graphene nanoplatelets-silver nanorods-polymer based in situ hybrid electrode for electroanalysis of dopamine and ascorbic acid in biological samples, Appl. Surf. Sci., 2018, 449, 558-566.

6 L. Q. Xie, Y. H. Zhang, F. Gao, Q. A. Wu, P. Y. Xu, S. S. Wang, N. N. Gao and Q. X. Wang, A highly sensitive dopamine sensor based on a polyaniline/reduced graphene oxide/ Nafion nanocomposite, Chin. Chem. Lett., 2017, 28, 41-48.

7 P. Wiench, Z. González, R. Menéndez, B. Grzyb and G. Gryglewicz, Beneficial impact of oxygen on the electrochemical performance of dopamine sensors based on N-doped reduced graphene oxides, Sens. Actuators, B, 2018, 257, 143-153.

8 A. Ejaz, Y. Joo and S. Jeon, Fabrication of 1,4-bis(aminomethyl) benzene and cobalt hydroxide @graphene oxide for selective detection of dopamine in the presence of ascorbic acid and serotonin, Sens. Actuators, B, 2017, 240, 297-307.

9 Y. Haldorai, A. T. E. Vilian, M. Rethinasabapathy, Y. S. Huh and Y. K. Han, Electrochemical determination of dopamine using a glassy carbon electrode modified with 
TiN-reduced graphene oxide nanocomposite, Sens. Actuators, $B, 2017,247,61-69$.

10 X. Liu, E. Shangguan, J. Li, S. Ning, L. Guo and Q. Li, A novel electrochemical sensor based on FeS anchored reduced graphene oxide nanosheets for simultaneous determination of dopamine and acetaminophen, Mater. Sci. Eng. C, 2017, 70, 628-636.

11 Y. Li, Y. Gu, B. Zheng, L. Luo, C. Li, X. Yan, T. Zhang, N. Lu and Z. Zhang, A novel electrochemical biomimetic sensor based on poly(Cu-AMT) with reduced graphene oxide for ultrasensitive detection of dopamine, Talanta, 2017, 162, 80-89.

12 A. T. E. Vilian, S. An, S. R. Choe, C. H. Kwak, Y. S. Huh, J. Lee and Y. K. Han, Fabrication of 3D honeycomb-like porous polyurethane-functionalized reduced graphene oxide for detection of dopamine, Biosens. Bioelectron., 2017, 86, 122128.

13 E. Er, H. Celikkan and N. Erk, A novel electrochemical nanoplatform based on graphene/platinum nanoparticles/Nafion composites for the electrochemical sensing of metoprolol, Sens. Actuators, B, 2017, 238, 779-787.

14 K. Zhang, X. Chen, Z. Li, Y. Wang, S. Sun, L. Wang, T. Guo, D. Zhang, Z. Xue, X. Zhou and X. Lu, Au-Pt bimetallic nanoparticles decorated on sulfonated nitrogen sulfur codoped graphene for simultaneous determination of dopamine and uric acid, Talanta, 2018, 178, 315-323.

15 N. S. Anuar, W. J. Basirun, M. Ladan, M. Shalauddin and M. S. Mehmood, Fabrication of platinum nitrogen-doped graphene nanocomposite modified electrode for the electrochemical detection of acetaminophen, Sens. Actuators, B, 2018, 266, 375-383.

16 R. M. A. Hameed and S. S. Medany, Evaluation of core-shell structured cobalt@platinum nanoparticles-decorated graphene for nitrite sensing, Synth. Met., 2019, 247, 67-80.

17 A. Zhang, X. Li and Y. He, Platinum/nitrogen-doped carbon nanoparticles synthesized in nitrogen-doped carbon quantum dots aqueous solution for methanol electrooxidation, Electrochim. Acta, 2016, 213, 332-340.

18 A. Arvinte, I. A. Crudu, F. Doroftei, D. Timpu and M. Pinteala, Electrochemical codeposition of silver-gold nanoparticles on CNT-based electrode and their performance in electrocatalysis of dopamine, J. Electroanal. Chem., 2018, 829, 184-193.

19 A. Deepi, G. Srikesh and A. S. Nesaraj, One pot reflux synthesis of reduced graphene oxide decorated with silver/ cobalt oxide: a novel nano composite material for high capacitance applications, Ceram. Int., 2018, 44, 2052420530.

20 D. Kim, S. Lee and Y. Piao, Electrochemical determination of dopamine and acetaminophen using activated grapheneNafion modified glassy carbon electrode, J. Electroanal. Chem., 2017, 794, 221-228.

21 S. Phetsang, J. Jakmunee, P. Mungkornasawakul, R. Laocharoensuk and K. Ounnunkad, Sensitive amperometric biosensors for detection of glucose and cholesterol using a platinum/reduced graphene oxide/ poly(3-aminobenzoic acid) film-modified screen-printed carbon electrode, Bioelectrochem, 2019, 127, 125-135.

22 M. T. Tajabadi, W. J. Basirun, F. Lorestani, R. Zakaria, S. Baradaran, Y. M. Amin, M. R. Mahmoudian, M. Rezayi and $\mathrm{M}$. Sookhakian, A novel non-enzymatic glucose sensor based on $\mathrm{Cu}$ nanoparticle modified graphene sheets electrode, Electrochim. Acta, 2015, 151, 126-133.

23 R. P. Rocha, A. G. Goncalves, L. M. Pastrana-Martínez, B. C. Bordoni, O. S. G. P. Soares, J. J. M. Órfão, J. L. Faria, J. L. Figueiredo, A. M. T. Silva and M. F. R. Pereira, Nitrogen-doped graphene-based materials for advanced oxidation processes, Catal. Today, 2015, 249, 192-198.

24 H. Ghadimi, B. Nasiri-Tabrizi, P. M. Nia, W. J. Basirun, R. M. A. Tehrani and F. Lorestani, Nanocomposites of nitrogen-doped graphene decorated with a palladium silver bimetallic alloy for use as a biosensor for methotrexate detection, RSC Adv., 2015, 5, 99555-99565.

25 Y. V. M. Reddy, B. Sravani, D. M. Fernandes, C. Madhuri, L. S. Sarma and G. Madhavi, Facile one pot synthesis of bimetallic $\mathrm{Pd}-\mathrm{Ag} /$ reduced graphene oxide nanocomposite as an electrochemical sensor for sensitive detection of antihypotensive drug, Colloids Surf., A, 2018, 546, 293-300.

26 N. Hao, R. Hua, S. Chen, Y. Zhang, Z. Zhou, J. Qian, Q. Liu and $\mathrm{K}$. Wang, Multiple signal-amplification via $\mathrm{Ag}$ and $\mathrm{TiO}_{2}$ decorated 3D nitrogen doped graphene hydrogel for fabricating sensitive label-free photoelectrochemical thrombin aptasensor, Biosens. Bioelectron., 2018, 101, 14-20.

27 S. Manchala, L. R. Nagappagari, S. M. Venkatakrishnan and V. Shanker, Solar-light harvesting bimetallic Ag/Au decorated graphene plasmonic system with efficient photoelectrochemical performance for the enhanced water reduction process, ACS Appl. Nano Mater., 2019, 2(8), 47824792.

28 Y. Zhao, L. Chen and Y. Meng, The fabrication of Pt/Co nanocomposite supported on reduced graphene oxide for methanol oxidation, Int. J. Electrochem. Sci., 2019, 14, 6826-6839.

29 V. Vinoth, J. J. Wu, A. M. Asiri and S. Anandana, Sonochemical synthesis of silver nanoparticles anchored reduced graphene oxide nanosheets for selective and sensitive detection of glutathione, Ultrason. Sonochem., 2017, 39, 363-373.

30 M. Shalauddin, S. Akhter, W. J. Basirun, S. Bagheri, N. S. Anuar and M. R. Johan, Hybrid nanocellulose/fMWCNTs nanocomposite for the electrochemical sensing of diclofenac sodium in pharmaceutical drugs and biological fluids, Electrochim. Acta, 2019, 304, 323-333.

31 Y. Cheng, H. Li, C. Fang, L. Ai, J. Chen, J. Su, Q. Zhang and Q. Fu, Facile synthesis of reduced graphene oxide/silver nanoparticles composites and their application for detecting heavy metal ions, J. Alloys Compd., 2019, 787, 683-693.

32 S. Kumar, M. Mahajan, R. Singh and A. Mahajan, Silver nanoparticles anchored reduced graphene oxide for enhanced electrocatalytic activity towards methanol oxidation, Chem. Phys. Lett., 2018, 693, 23-27. 
33 S. Akhter, W. J. Basirun, Y. Alias, M. R. Johan, S. Bagheri, M. Shalauddin, M. Ladan and N. S. Anuar, Enhanced amperometric detection of paracetamol by immobilized cobalt ion on functionalized MWCNTs-chitosan thin film, Anal. Biochem., 2018, 551, 29-36.

$34 \mathrm{H}$. Devnani, S. Ansari, S. P. Satsangee and R. Jain, $\mathrm{ZrO}_{2}$ Graphene-Chitosan nanocomposite modified carbon paste sensor for sensitive and selective determination of dopamine, Mater. Today Chem., 2017, 4, 17-25.

35 M. M. Rahman, N. S. Lopa, M. J. Ju and J. J. Lee, Highly sensitive and simultaneous detection of dopamine and uric acid at graphene nanoplatelet-modified fluorine-doped tin oxide electrode in the presence of ascorbic acid, $J$. Electroanal. Chem., 2017, 792, 54-60.

36 M. Cheng, X. Zhang, M. Wang, H. Huang and J. Ma, A facile electrochemical sensor based on well-dispersed graphenemolybdenum disulfide modified electrode for highly sensitive detection of dopamine, J. Electroanal. Chem., 2017, 786, 1-7.

37 Q. Zhu, J. Bao, D. Huo, M. Yang, C. Hou, J. Guo, M. Chen, H. Fa, X. Luo and Y. Ma, 3D Graphene hydrogel-gold nanoparticles nanocomposite modified glassy carbon electrode for the simultaneous determination of ascorbic acid, dopamine and uric acid, Sens. Actuators, B, 2017, 238, 1316-1323.

38 T. K. Aparna, R. Sivasubramanian and M. A. Dar, One-pot synthesis of $\mathrm{Au}-\mathrm{Cu}_{2} \mathrm{O} / \mathrm{rGO}$ nanocomposite based electrochemical sensor for selective and simultaneous detection of dopamine and uric acid, J. Alloys Compd., 2018, 741, 1130-1141.
39 A. Khoshroo, L. Hosseinzadeh, A. Sobhani-Nasab, M. RahimiNasrabadi and H. Ehrlich, Development of electrochemical sensor for sensitive determination of oxazepam based on silver-platinum core-shell nanoparticles supported on graphene, J. Electroanal. Chem., 2018, 823, 61-66.

40 G. Xu, Z. A. Jarjes, V. Desprez, P. A. Kilmartin and J. TravasSejdic, Sensitive, selective, disposable electrochemical dopamine sensor based on PEDOT-modified laser scribed graphene, Biosens. Bioelectron., 2018, 107, 184-191.

41 H. Wang, L. G. Xiao, X. F. Chu, Y. D. Chi and X. T. Yang, Rational design of gold nanoparticle/graphene hybrids for simultaneous electrochemical determination of ascorbic acid, dopamine and uric acid, Chin. J. Anal. Chem., 2016, 44(12), 1617-1625.

42 C. Zou, J. Zhong, S. Li, H. Wang, J. Wang, B. Yan and Y. Du, Fabrication of reduced graphene oxide-bimetallic PdAu nanocomposites for the electrochemical determination of ascorbic acid, dopamine, uric acid and rutin, J. Electroanal. Chem., 2017, 805, 110-119.

43 Y. Wang, Y. Huang, B. Wang, T. Fang, J. Chen and C. Liang, Three-dimensional porous graphene for simultaneous detection of dopamine and uric acid in the presence of ascorbic acid, J. Electroanal. Chem., 2016, 782, 76-83.

44 X. Ding, J. Bai, T. Xu, C. Li, H. M. Zhang and L. Qu, A novel nitrogen-doped graphene fiber microelectrode with ultrahigh sensitivity for the detection of dopamine, Electrochem. Commun., 2016, 72, 122-125.

45 D. Wang, F. Xu, J. Hu and M. Lin, Phytic acid/graphene oxide nanocomposites modified electrode for electrochemical sensing of dopamine, Mater. Sci. Eng. C, 2017, 71, 1086-1089. 